\title{
Measuring the Stellar Halo Velocity Anisotropy With 3D Kinematics
}

\author{
Emily C. Cunningham ${ }^{1}$, Alis J. Deason ${ }^{1},{ }^{2}$, Puragra Guhathakurta ${ }^{1}$, \\ Constance M. Rockosi ${ }^{1}$, Roeland P. van der Marel ${ }^{3}$, S. Tony Sohn ${ }^{4}$ \\ ${ }^{1}$ Department of Astronomy \& Astrophysics, UC Santa Cruz \\ 1156 High Street, Santa Cruz, CA 95060, USA \\ 2 Physics Department, Stanford University \\ 382 Via Pueblo Rd, Stanford, CA 94305, USA \\ ${ }^{3}$ Space Telescope Science Institute \\ 3700 San Martin Drive, Baltimore, MD 21218, USA \\ ${ }^{4}$ Department of Physics and Astronomy, The Johns Hopkins University \\ Baltimore, MD 21218, USA
}

\begin{abstract}
We present the first measurement of the anisotropy parameter $\beta$ using 3D kinematic information outside of the solar neighborhood. Our sample consists of 13 Milky Way halo stars with measured proper motions and radial velocities in the line of sight of M31. Proper motions were measured using deep, multi-epoch HST imaging, and radial velocities were measured from Keck II/DEIMOS spectra. We measure $\beta=-0.3_{-0.9}^{+0.4}$, which is consistent with isotropy, and inconsistent with measurements in the solar neighborhood. We suggest that this may be the kinematic signature of a relatively early, massive accretion event, or perhaps several such events.
\end{abstract}

Global kinematic properties, such as the relative pressure between tangential and radial velocity components, otherwise known as the velocity anisotropy, can provide important insight into the formation of the stellar halo. Using the 6D phase space information from our survey, we have made the first direct measure of the velocity anisotropy outside of the solar neighborhood (Cunningham et al. 2016).

In Deason et al. (2013b), we published the first proper motions of distant Milky Way halo stars; in Cunningham et al. (2016), we present the line-of-sight (LOS) velocities for those same stars. Our 13 objects are the first stars outside of the solar neighborhood to have 3D kinematic information. The left panel of Figure 1 shows a cumulative LOS velocity histogram of the 13 stars in our sample, and the right panel shows the proper motions of the halo stars color coded by LOS velocity (velocities are shown in the Galactocentric frame). We estimated the parameters of the velocity ellipsoid $\left(\left\langle v_{l}\right\rangle, \sigma_{\mathrm{LOS}}, \sigma_{l}, \sigma_{b}\right)$ using Markov Chain Monte Carlo (Figure 2), and made the first measurement of the anisotropy parameter $\beta$ using 3D kinematic information outside of the solar neighborhood. We find $\beta=-0.3_{-0.9}^{+0.4}$, consistent with isotropy and inconsistent with solar neighborhood measurements, which find a highly radial anisotropy $(\beta \sim 0.5-0.7)$. Simulations of accreted stellar haloes indicate that $\beta$ should increase outward (e.g. Abadi et al. 2006), and yet we observe a "dip" in the radial anisotropy profile. This dip is coincident with the break radius in the Milky Way's stellar density profile. We argue that the presence of large, dynamically "fluffy" global substructre, such as a shell (or multiple shells), is one explanation for both the steep fall-off in stellar density beyond the break radius and the decrease in anisotropy at that radius. Deason et al. (2013a) argued that a break in the Milky Way stellar density profile could be created by the build-up of stars at apocenter from either one relatively massive accretion event or several, synchronous accretion events. In this 

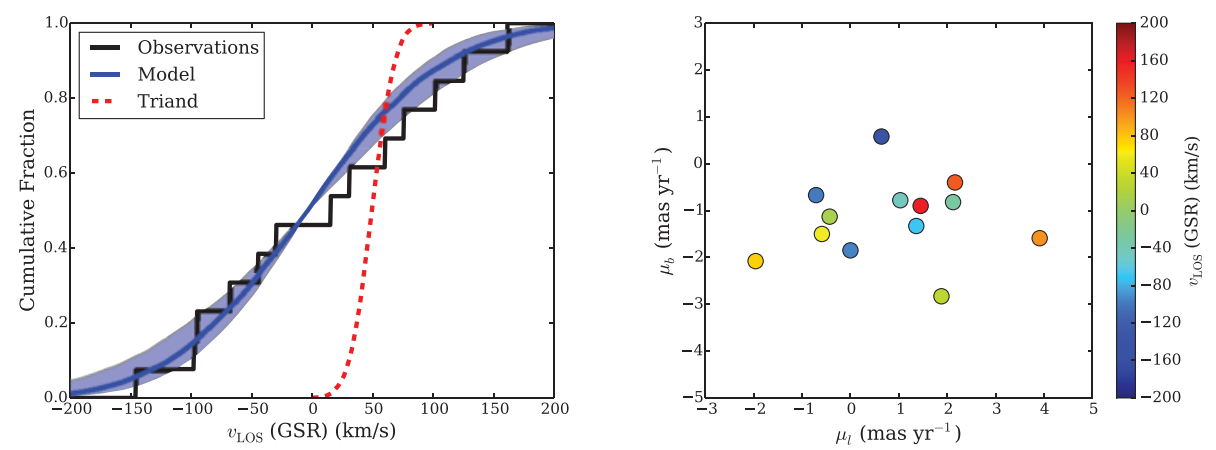

Figure 1. Left: Cumulative histogram of LOS velocities of the 13 halo stars in our sample (black). The overplotted blue line shows the cumulative distribution function (CDF) for the most likely value for $\sigma_{\mathrm{LOS}}$, with the shaded blue region indicating the $68 \%$ confidence region. An approximate CDF for TriAnd is shown in red $\left(v_{0} \sim 50 \mathrm{~km} \mathrm{~s}^{-1}, \sigma \sim 15 \mathrm{~km} \mathrm{~s}^{-1}\right)$. Right: Proper motions in galactic coordinates of our 13 halo stars, color coded by LOS velocity relative to the Galactic Standard of Rest (GSR).

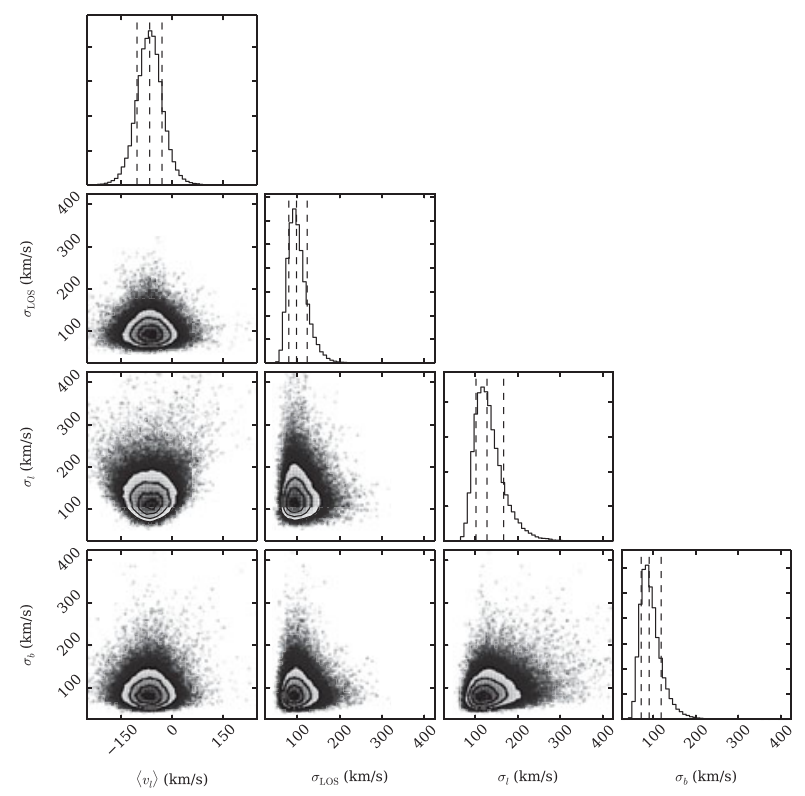

Figure 2. Projections of the posterior probability distribution for the four free parameters of the velocity ellipsoid in our model: $\left\langle v_{l}\right\rangle, \sigma_{\mathrm{LOS}}, \sigma_{l}$ and $\sigma_{b}$. Contours are shown at $0.5,1,1.5$ and $2 \sigma$, respectively. The top panel in each column shows the 1D marginalized PDF for each parameter, with $0.16,0.5$ and 0.84 quantiles indicated by dashed vertical lines.

scenario, we would expect the stars to have an increase in tangential motion relative to radial motion at the turnaround radius, and thus a more isotropic $\beta$, just as we observe.

\section{References}

Abadi, M.G., Navarro, J.F., \& Steinmetz, M.2006, MNRAS, 365, 747

Cunningham, E. C., Deason, A. J., Guhathakurta, P., et al. 2016, arXiv:1602.03180

Deason, A. J., Belokurov, V., Evans, N. W., \& Johnston, K. V. 2013a, ApJ, 763, 113

Deason, A. J., Van der Marel, R. P., Guhathakurta, P., Sohn, S. T., \& Brown, T. M. 2013b, ApJ, 766, 24 